\title{
Effects of Postharvest Partial Dehydration and Prolonged Treatments with Ethylene on Transcript Profiling in Skins of Wine Grape Berries
}

\author{
F.M. Rizzini and C. Bonghi \\ Department of Environmental \\ Agronomy and Crop Science \\ University of Padova \\ Viale dell' Università 16 \\ 35025 Legnaro (Padova) \\ Italy
}

\author{
L. Chkaiban, F. Martinelli and P. Tonutti \\ Scuola Superiore Sant'Anna \\ Piazza Martiri della Libertà 33 \\ 56127 Pisa \\ Italy
}

Keywords: gene expression, genomics, microarray, polyphenols, secondary metabolism, transcriptome, Vitis vinifera

\begin{abstract}
For certain food products, postharvest controlled stresses or treatments with specific elicitors are applied to induce desired physical/chemical changes and/or to positively affect phytochemical content. This is the case of wine grapes where both strategies, singularly applied or coupled, can be used to modulate berry composition and, as a consequence, affect wine quality traits. Since the knowledge of the effects of these postharvest treatments on berry metabolism and the regulation of gene expression is very limited, a large-scale transcriptome analysis has been carried out, using an oligo-based microarray (14,562 probes) on skins of wine grape (Vitis vinifera L.) berries subjected to dehydration, at different rates, up to $30 \%$ of weight loss or to ethylene treatment $(500 \mathrm{ppm}$ for 7 days) after harvest. A number of differentially expressed targets was detected following both treatments, indicating that grape berries are still reactive at advanced stages of postharvest dehydration and that ethylene induces marked changes in transcriptome after harvest also in non-climacteric fruit such as grape berries.
\end{abstract}

\section{INTRODUCTION}

Postharvest dehydration of grape berries is applied for raisin production and for making wines (often called dessert wines) that are characterized, as result of both concentration process and metabolic changes occurring in berry flesh and skin, by high sugar and/or alcohol content and peculiar taste and aroma. The rate and the intensity of postharvest berry dehydration, often reaching $40-50 \%$ of weight loss (WL), markedly affect the resulting wines and this is due, primarily but not exclusively, to differences in terms of sugar and organic acid content of flesh cells. Following berry dehydration, sugar concentration increases whereas organic acid content tends to remain stable (or slightly reduced) due to increments of tartaric acid (concentration effects) and decreases of malic acid that is likely consumed as substrate for respiration. Costantini et al. (2006) report an increase of $\mathrm{CO}_{2}$ production in wine grape ('Malvasia') berries undergoing postharvest dehydration starting from a WL of $10 \%$ and reaching the highest values around $22 \%$ WL. Bellincontro et al. (2004) suggest that a shift from aerobic to anaerobic metabolism occurs when grape berries lose about $10-15 \%$ of fresh weight. As consequence of concentration and increased respiration rate, sugar/organic acid and tartaric/malic acid ratios increase whereas glucose/fructose ratio decreases. Water loss from detached fruit may induce marked changes also in terms of secondary metabolism (Kays and Paull, 2004): limited water stress (1-3\% of WL), induce increases in abscissic acid (ABA) and ethylene biosynthesis, and pectolitic (e.g., polygalacturonase) enzyme activities. At higher dehydration rate (3-5\%), loss of membrane integrity, altered volatile pattern and phenol metabolism occur.

Considering specifically grape berry, limited information is available on metabolic aspects induced by postharvest dehydration. Costantini et al. (2006) observed, in 
'Malvasia' berries, increases of ABA content and lipoxygenase (LOX) activity in correspondence of a weight loss of about $11 \%$. These metabolic changes are accompanied by a rise of C6 compounds (hexen-1-ol, hexanal and (E)-hex-2-enal) and proline content. A more detailed study on volatile pattern following berry dehydration of three different grape varieties ('Trebbiano', 'Malvasia' and 'Sangiovese') has been carried out by Bellincontro et al. (2004). Results indicate that water loss greatly affects the volatile production and that the dehydration rate modifies the range of volatiles released. Similarly, total phenols and anthocyanin content appeared affected by the intensity and rate of dehydration. Taken together these data indicate a marked effect of postharvest water loss on secondary metabolism of grape berry and, in particular, of the skins that are metabolically active throughout fruit development and ripening and synthesize the main compounds (polyphenols, aromas) responsible for wine quality. A paper by Deytieux et al. (2007) confirms, using a proteomic approach, that significant changes in primary and secondary metabolism occur in grape berry skin during the transition from immature (veraison stage) to mature stage.

Although grape berries are classified as non-climacteric fruit, ethylene plays a regulatory role at the onset (veraison) of ripening. Applications of 2-CEPA, an ethylenereleasing compound, or ethylene at veraison induce an increase of anthocyanins (ElKereamy et al., 2003) and berry size (Chervin et al., 2008) by up-regulating expression of genes involved in the polyphenol biosynthesis and cell wall extensibility, respectively. The positive effects of ethylene on berry size are in accordance with the reduction of the berry expansion induced by 1-methylcyclopropene (1-MCP) application, an inhibitor of ethylene action (Chervin et al., 2004). No information is available on the effects of ethylene application on detached grape berries.

Gene expression studies are crucial for better elucidating complex biological processes and the basic mechanisms regulating responses to different stresses and environmental factors. In this context, genomics tools and large-scale transcript analysis based on microarrays have been particularly useful to better understand the fruit ripening process in several species including grape (Terrier et al., 2005), and the effect of postharvest treatments and/or storage conditions (Ponce-Valadez et al., 2009).

The aim of the present paper was that of analyzing transcriptome profile changes in skins of wine grape berries allowed to lose water, at different rate, up to $30 \%$ of WL, and following postharvest ethylene treatment.

\section{MATERIALS AND METHODS}

\section{Plant Material and Treatments}

Grapes (Vitis vinifera L. 'Raboso Piave') were harvested at technical maturity and selected bunches were placed in 24 perforated plastic boxes (6 per treatment, $6.5 \mathrm{~kg}$ per box). Dehydration was induced under these two following conditions:

a) Uncontrolled (slow dehydration rate, S). Bunches were stored in a traditional naturally ventilated storage room up to 90 days. Under these conditions RH ranged between 67 and $96 \%$ and temperature between 5 and $18^{\circ} \mathrm{C}$;

b) Controlled (rapid dehydration rate, R). Bunches were stored up to 12 days in a dehydration tunnel (Marvil Engineering SpA, Egna-Ora, Bolzano, Italy) where the environmental conditions were set as follows: temperature $20 \pm 1^{\circ} \mathrm{C}, \mathrm{RH} 40 \pm 5 \%$, air flow: $1-1.5 \mathrm{~m} / \mathrm{s}$.

Weight loss was monitored by weighing 10 representative bunches. At harvest (T0) and at 10 and 30\% weight loss (WL) following both slow (S10 and S30) and rapid (R10 and R30) dehydration, selected berries of similar size and from the central part of the bunches were detached, hand-peeled, and the skins immediately frozen in liquid nitrogen and stored at $-80^{\circ} \mathrm{C}$.

For ethylene treatment, selected bunches were placed in plastic boxes and ethylene $(500 \mathrm{ppm})$ in humidified air stream was fluxed at the rate of $1.5 \mathrm{~L} / \mathrm{h}$ for 7 days. At the end of the treatment berries of similar size were detached, hand-peeled, and the skins 
immediately frozen in liquid nitrogen and stored at $-80^{\circ} \mathrm{C}$.

\section{Microarray Analysis}

Microarray chips were constructed by spotting the Grape Genome Oligo Set Version 1.0 (Operon) containing 14,562 probes (70mers) representing an equal number of open reading frames (ORFs) from Vitis vinifera. Total RNA $(20 \mu \mathrm{g})$, extracted from skins of dehydrated berries at different rate and intensity (S10, S30, R10 and R30) and from ethylene-treated and untreated grape berries as described by Rizzini et al. (2009), was converted into target cDNA by reverse transcription using the SuperScriptTM Indirect cDNA Labeling System (Invitrogen). cDNA labelling, microarray hybridization and data analysis, including functional categorization, were performed as described by Trainotti et al. (2006) and Rizzini et al. (2009).

\section{RESULTS AND DISCUSSION}

Microarray hybridizations were performed comparing each dehydrated sample (S10, S30, R10 and R30) with samples collected at harvest (T0). At 10\% FW dehydration, a total of 84 and 68 targets resulted as differentially expressed following slow (S10) and rapid (R10) dehydration, respectively, whereas this number dramatically increased when $30 \%$ FW was lost: in fact, 309 and 262 differentially expressed targets were detected in $\mathrm{S} 30$ and $\mathrm{R} 30$ samples, respectively.

Functional characterization of differentially expressed genes highlights that, independently of the rate and intensity of dehydration, differential expression was present in particular for genes associated to general metabolism (01), regulatory processes (16) and responses to biotic and abiotic stimuli $(32,34$ and 36). In all these functional categories, the number of targets differentially expressed increased, following both slow and rapid dehydration rate, when WL shifted from 10 to $30 \%$ (Fig. 1A and B). This clearly indicates that grape berries (skins) are still reactive (at least at transcriptional level) at advanced stages of postharvest dehydration. The rate of dehydration appeared to be effective in modulating the pattern of gene expression, as demonstrated in Figure 1C and $\mathrm{D}$, where the number of induced targets belonging to functional categories $01,16,32$ and 34 resulted higher in both 10 and 30\% WL samples if the dehydration process was slow. These data confirm the importance of applying appropriate environmental conditions during dehydration to modulate the water flux from the inside of the berry to the surrounding environment.

If bunches are stored in dehydration tunnels, gaseous treatments aimed at affecting berry metabolism and composition could be applied. A preliminary experiment has been carried out to evaluate, at transcriptomic level, the effect of a prolonged ( 7 days) treatment of ethylene at the concentration of $500 \mathrm{ppm}$. The effects of such treatment on skin transcriptome is evident analyzing microarray hybridization data. In fact, 139 genes resulted up-regulated by the gaseous hormone whereas 16 appeared repressed. Considering the putative function of these gene differentially expressed following the hormone treatment, some of them appear to be involved in aromatic compound and secondary metabolism. In particular, an up-regulation of specific genes involved in phenylpropanoid biosynthetic pathway (phenylalanine ammonia lyase, chalcone synthase, flavonone 3-hydroxylase, dihydroflavonol 4- reductase, MybA) has been observed (Fig. 2). No effects were observed on UDP-glucose:flavonoid 3-O-glucosyltransferase (UFGT) gene transcription, differently from the findings of El-Kereamy et al. (2003) who observed an up-regulation induced by the hormone at veraison. This result suggests that the UFTG is differentially regulated during fruit development and ripening. It is interesting also to notice the up-regulation induced by the hormone of three glutathioneS-transferase (GST) genes (Fig. 2). Glutathione has been shown to have a prodigious number of critical roles in plants linked by essential signalling functions that integrate plant growth, development and defence processes (Maughan and Foyer, 2006). Glutathione is a powerful reducing agent and is a key player in the plant redox-state (Noctor et al. 2002). In addition, it has been demonstrated that one GST gene performs 
the last genetically defined step of the maize anthocyanin pigment pathway, the conjugation of glutathione to cyanidin 3-glucoside that is then transported to the vacuole via a tonoplast Mg-ATP-requiring glutathione pump (Alfenito et al., 1998). Since stable anthocyanin pigmentation occurs when the molecules are transferred to the vacuole, it could be hypothesized that these processes also occur in grape cells with ethylene playing a regulatory role. Among up-regulated targets by ethylene treatment, some corresponding to cell wall hydrolases have also been identified. Considering their role in cell wall structure and architecture, these results could be related to the increased extractability of polyphenols induced by ethylene (data not shown).

Results indicate that, following postharvest ethylene treatment, Vitis vinifera berries show marked changes in transcriptome profiles with marked consequences in skin composition and, probably, in the characteristics of the resulting wines. Genes involved in the polyphenol biosynthetic pathway appear to react differently to exogenous ethylene treatment: its effect appears to be variable in relation to the duration of the incubation period.

\section{Literature Cited}

Alfenito, M.R., Souer, E., Goodman, C.D., Buell, R., Mol, J., Koes, R. and Walbot, V. 1998. Functional complemementation of anthocyanin sequestration in the vacuole by widely divergent glutathione-S-transferase. Plant Cell 10:1135-1150.

Bellincontro, A., De Santis, D., Botondi, R., Villa, I. and Mencarelli, F. 2004. Different postharvest dehydration rates affect quality characteristics and volatile compounds of 'Malvasia', 'Trebbiano' and 'Sangiovese' grapes for wine production. J. Sci. Food Agric. 84:1791-1800.

Chervin, C., El-Kereamy, A., Roustan, J.P., Latché, A., Lamon, J. and Bouzayen, M. 2004. Ethylene seems required for the berry development and ripening in grape, a non-climacteric fruit. Plant Sci. 167:1301-1305.

Chervin, C., Tira-umphon, A., Terrier, N., Zouine, M., Severac, D. and Roustan, J.-P. 2008. Stimulation of the grape berry expansion by ethylene and effects on related gene transcriptis, over the ripening phase. Physiol. Plant. 134:534-546

Costantini, V., Bellincontro, A., De Santis, D., Rotondi, R. and Mencarelli, F. 2006. Metabolic changes of 'Malvasia' grapes for wine production during postharvest drying. J. Agric. Food Chem. 54:3334-3340.

Deytieux, C., Geny, L., Lapaillerie, D., Claverol, S., Bonneu, M. and Doneche, B. 2007. Proteome analysis of grape skin during ripening. J. Exp. Bot. 58:1851-1862.

El-Kereamy, A., Chervin, C., Roustan, J.P., Cheynier, V., Souquet, J., Moutounet, M., Raynal, J.M., Ford, C., Latchè, A., Pech, J.P. and Bouzayen, M. 2003. Exogenous ethylene stimulates the long-term expression of genes related to anthocyanin biosynthesis in grape berries. Physiol. Plant. 119:175-182.

Kays, S.J. and Paull, R.E. (eds.). 2004. Postharvest Biology. Exon Press, Athens Georgia, USA.

Maughan, S. and Foyer, C.H. 2006. Engineering and genetic approaches to modulating the glutathione network in plants. Physiol. Plant.126:382-397.

Noctor, G., Gomez, L., Vanacker, H. and Foyer, C.H. 2002. Glutathione homeostasis and signaling: the influence of biosynthesis, compartmentation and transport. J. Exp. Bot. 53:1283-1304

Ponce-Valadez, M., Moore, S., Giovannoni, J.J., Gan, S. and Watkins, C.B. 2009. Differential fruit gene expression in two strawberry cultivars in response to elevated $\mathrm{CO}_{2}$ during storage revealed by a heterologous fruit microarray approach. Postharvest Biol. Technol. 51:131-140.

Rizzini, F.M., Bonghi, C. and Tonutti, P. 2009. Postharvest water loss induces marked changes in transcript profiling in skins of wine grape berries. Postharvest Biol. Technol. 52:247-253

Terrier, N., Glissant, D., Grimplet, J., Barrieu, F., Abbal, P., Couture, C., Ageorges, A., Atanassova, R., Leon, C., Renaudin, J.P., Dedaldechamp, F., Romieu, C., Delrot, S. 
and Hamdi, S. 2005. Isogene specific oligo arrays reveal multifaceted changes in gene expression during grape berry (Vitis vinifera L.) development. Planta 222:832-847.

Trainotti, L., Bonghi, C., Ziliotto, F., Zanin, D., Rasori, A., Casadoro, G., Ramina, A. and Tonutti, P. 2006. The use of microarray $\mu$ PEACH1.0 to investigate transcriptome changes during transition from pre-climacteric to climacteric phase in peach fruit. Plant Sci. 170:606-613.

\section{Figures}
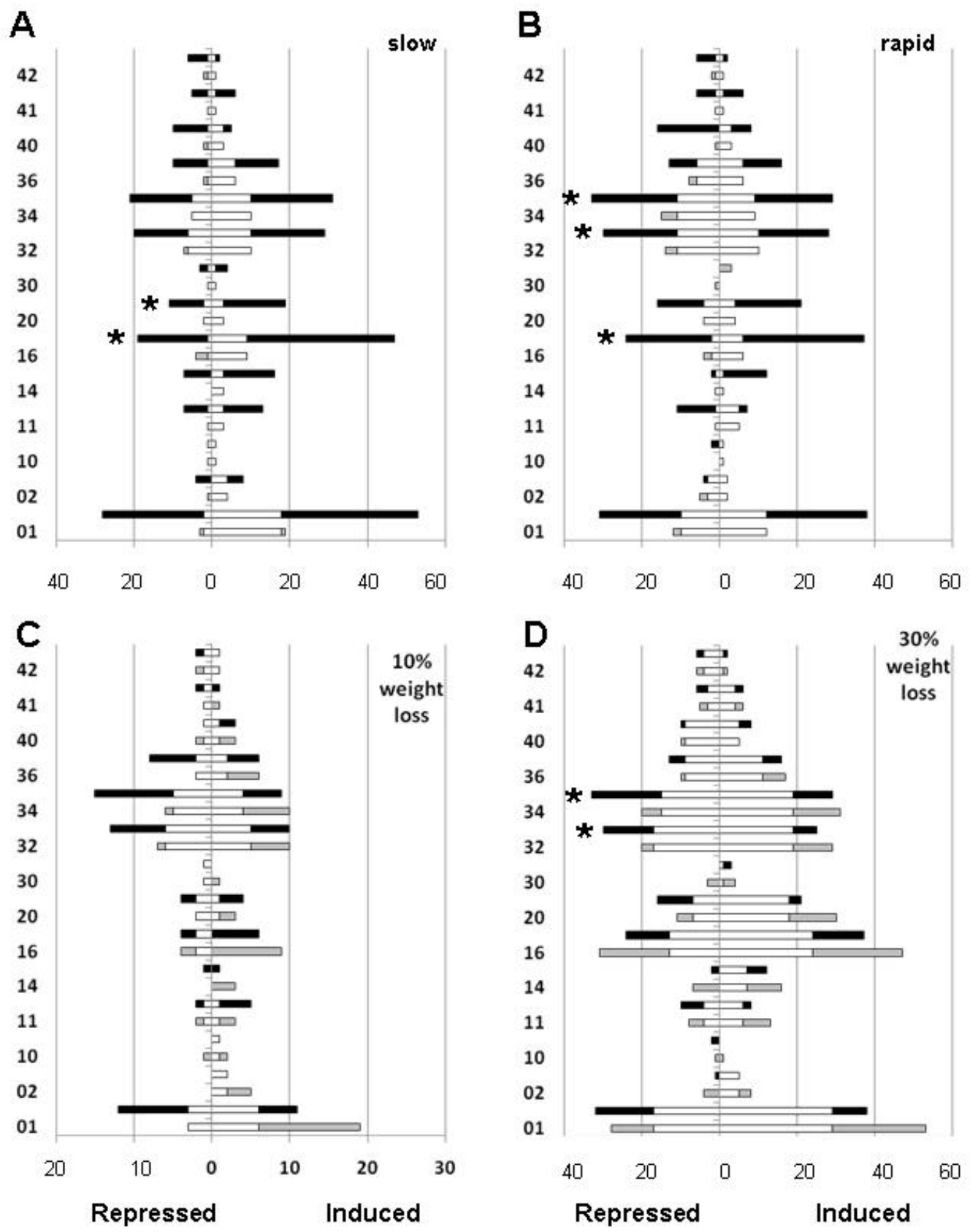

Number of probes

Fig. 1. Caption see next page. 
Fig. 1. Functional categorization, based on MIPS platform, of induced (right side of each panel) or repressed (left side of each panel) probes spotted on the microarray considering water loss rate (slow [A] or rapid [B]) or water loss intensity $(10 \%[\mathrm{C}]$ and $30 \%[\mathrm{D}]$ ). For each functional category (indicated with a number on the $y$ axis) two bars are reported. In panels A and B the lower and the upper bars represent the number of repressed (left) and induced (right) probes after 10 and $30 \%$ of WL, respectively. Common probes are represented by white bars, while those specific for 10 and $30 \% \mathrm{WL}$ are indicated by grey and black bars, respectively. In panels $C$ and $D$ the lower and the upper bars represent the number of repressed (left) and induced (right) probes following slow and rapid dehydration, respectively. Common probes are represented by white bars, while those specific for slow and rapid dehydration are indicated by grey and black bars, respectively. Functional categories are (a) Metabolism (01 Metabolism; 02 Energy); (b) Information pathways (10 Cell cycle and DNA processing, 11 Transcription, 14 Protein fate, 16 Protein with binding function or requirement); (c) Transport (20 Cellular transport, transport facilities and transport routes); (d) Perception and response to stimuli (30 Cellular communication/signal transduction mechanism, 32 Cell rescue, defense and virulence, 34 Interaction with the environment, 36 Systemic interaction with the environment); (e) Developmental process (40 Cell fate, 41 Development (systemic) 42 Biogenesis of cellular components). Asterisks indicate over-represented categories at $\mathrm{p}$ value of 0.05 .

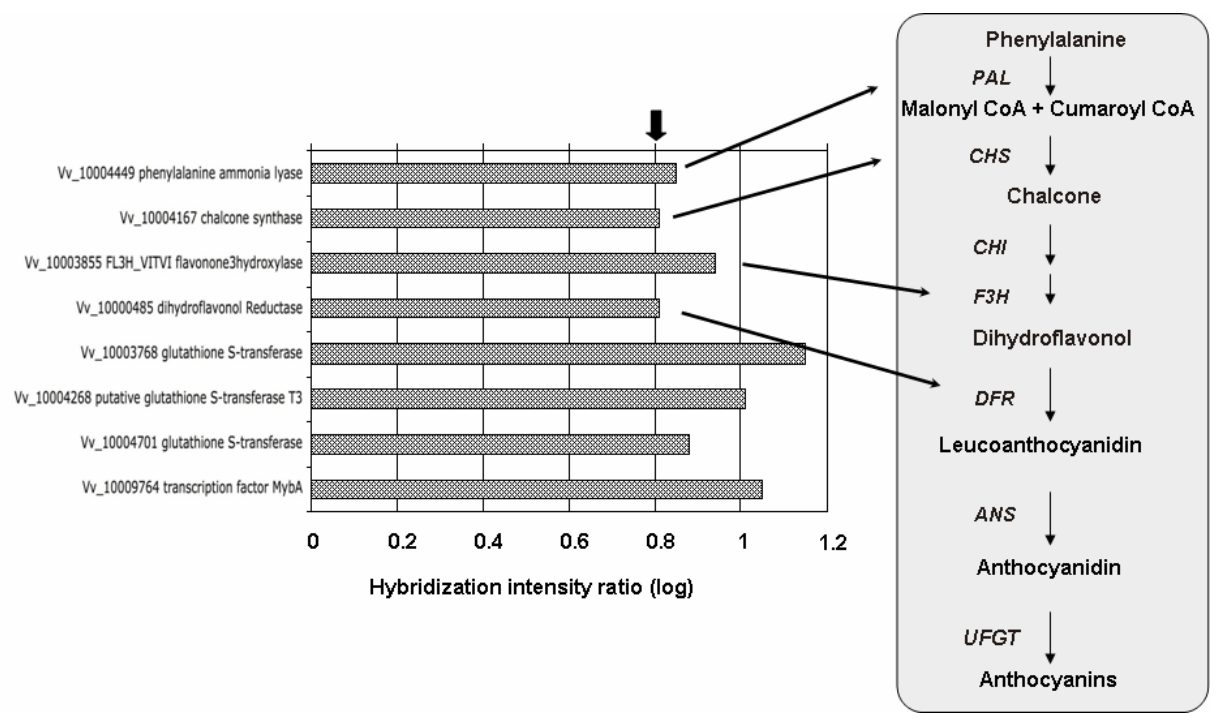

Fig. 2. Microarray hybridization intensity ratio (ethylene treated/untreated samples) of targets corresponding to genes involved in phenylpropanoid metabolism (a simplified scheme of the biosynthetic pathway is reported in the inset). Data represent median value of 6 replicates. Arrow indicates ratio threshold used to identify genes up-regulated ( $>0.8 \log$ of hybridization intensity ratio). 